\title{
Use of the ductal curvature index to assess the risk of ductal stenting in patients with duct-dependent pulmonary circulation
}

\author{
Nathalie Mini^, Martin B. E. Schneider, Peter A. Zartner \\ Department of Cardiology, German Paediatric Heart Centre, University Hospital of Bonn, Bonn, Germany \\ Contributions: (I) Conception and design: All authors; (II) Administrative support: MBE Schneider; (III) Provision of study materials or patients: MBE \\ Schneider, PA Zartner; (IV) Collection and assembly of data: N Mini; (V) Data analysis and interpretation: All authors; (VI) Manuscript writing: All \\ authors; (VII) Final approval of manuscript: All authors. \\ Correspondence to: Nathalie Mini. Cardiac Catheterization Laboratory, Paediatric Heart Centre, University hospital of Bonn, Campus Venusberg 1, \\ 53721 Bonn, Germany. Email: Nathalie.Mini@ukbonn.de.
}

\begin{abstract}
Background: To evaluate whether a quantitative curvature threshold can contribute to risk stratification of ductal stenting in patients with a duct-dependent pulmonary circulation (DDPC).

Methods: A single-center retrospective analysis was performed. The ductal curvature index (DCI) was calculated in 71 patients with DDPC. The ducts were divided into four classes based on quartile thresholds of DCI: class I $(\leq 0.12)$, class II $(0.13-0.33)$, class III $(0.34-0.44)$, and class IV $(\geq 0.45)$. The primary outcome of this study was defined as free from all of the following: (I) intervention related death, (II) need of unplanned surgery (III) need of unplanned pulmonary valve (PV) perforation with stent in right ventricular outflow tract (RVOT), and (IV) intervention related permanent complications.

Results: Eighty percent of patients in class IV (DCI $\geq 0.45$ ) failed to achieve the primary outcome; odds ratio (OR) 9 and $95 \%$ confidence interval (CI): 3.7-21.4 compared to $26.6 \%$ in all classes with DCI $<0.45$. $66.7 \%$ of these patients needed unplanned surgery or PVP with RVOT stent; OR 12.4 (95\% CI: 4-39). 80\% of major complications were observed in class IV $(\mathrm{P}<0.01)$. Need of pulmonary arterioplasty was in class IV 53.3\%; OR 3.3 (95\% CI: 1.5-7.1).

Conclusions: DCI can be useful to guide the clinical decision-making in patients with torqued ducts. Patients with a DCI $\geq 0.45$ belong to a high-risk group, in which ductal stenting is associated with an elevated risk for early surgery or unplanned re-intervention.
\end{abstract}

Keywords: Pulmonary atresia; ductus arteriosus; ductal stenting; pulmonary perfusion and ductal curvature index

Submitted Jan 12, 2021. Accepted for publication Mar 15, 2021.

doi: 10.21037/tp-21-17

View this article at: http://dx.doi.org/10.21037/tp-21-17

\section{Introduction}

All patients with a duct-dependent pulmonary circulation (DDPC) need an adequate pulmonary perfusion for survival. Although surgical techniques have advanced significantly in recent years (1), some biventricular heart diseases with DDPC remain unamenable to be corrected in the neonatal period due to variable reasons (2-5). Such cardiac defects and all other DDPC with a single ventricle require a palliative procedure to ensure a sufficient pulmonary perfusion until the next planned operation. Either a surgical creation of an aorta- pulmonary shunt or an interventional PDA stenting will be required for bridging to the next surgery. Multiple studies in the last decades have demonstrated safety and efficacy of duct stenting as an alternative to a surgical creation of modified BlalockTaussig Shunt (mBT Shunt) (6-8). Stenting can spare

^ ORCID: 0000-0002-3527-3741. 


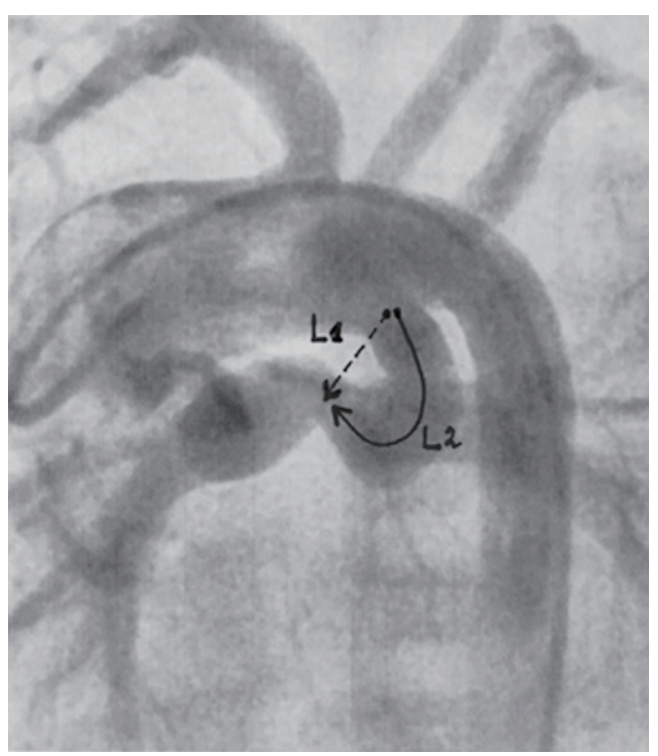

Figure 1 Demonstration of the two measured distances used to calculate the ductal curvature index. L1 represents the straight short distance between duct origin from aorta and duct insertion onto pulmonary arteries (PA). L2 represents the entire length of the duct between the aortic origin and the insertion of the duct onto PA.

patients the need for thoracotomy and reduce postoperative complications as well as asymmetrical development of pulmonary arteries. Additionally, it reduces the mortality rate and the need for extracorporeal membrane oxygenation (ECMO) (9-13). Despite the new developments in the field of coronary stents and the introduction of drug eluting stents that minimise in-stent stenosis and prolong the stent patency $(12,14)$, stenting of ducts with increased tortuosity ,especially when associated with pulmonary stenosis, is still challenging and fraught with risks (15-17).

Qureshi el al used a ductal classification scheme based on the tortuosity of the duct and found that patients with the highest tortuosity had increased the need of PDA stent reintervention and pulmonary arterioplasty (18). No studies to date, however, have identified a ductal morphology index with a clear-cut threshold that defines patients with high risk who may not respond favourably to stenting. Such a clinical index would be essential to provide a predive ability and guide the management decisions.

In search of a clearly defined threshold value that could mark the risk of an inappropriate PDA stent, we tried, as part of this retrospective cohort analysis, to first create a quantitative ductal morphology index and, second, to examine whether the risk for complications and/or unplanned re-interventions can be estimated through this particular threshold.

We present the study in accordance with the STROBE reporting checklist (available at: http://dx.doi.org/10.21037/ tp-21-17).

\section{Methods}

This is a single-center retrospective cohort analysis. All our consecutive patients with pulmonary atresia (PA) and DDPC undergoing PDA-stenting between Jan. 2011 and Oct. 2019 were recruited to evaluate the impact of their duct morphology on the stent outcome (diagnoses summarized in Table 1). Patients with PA and major aorta pulmonary collateral Arteries (MAPCAs) were excluded from the analysis as well as patients with pulmonary stenosis (PS) who have significant antegrade pulmonary perfusion.

The primary endpoint of this study was a composite outcome defined as free from all of following: (I) intervention related death, (II) need of unplanned surgery (III) need of unplanned PV-perforation (PVP) with RVOT-stent, and (IV) intervention related permanent complications. The occurrence of one or more of the endpoints was defined as an impaired outcome. DCI was calculated in all ducts using the following formula, which was adopted from a previous study (18): DCI $=(\mathrm{L} 2-\mathrm{L} 1)$ / L2, where L1 represents the straight short distance between duct origin from aorta and duct insertion onto pulmonary arteries. L2 represents the entire length of the duct between the aortic origin and the insertion of the duct onto pulmonary arteries (Figure 1). The two measurements were documented in millimetres. To optimize L2 measurement, especially in ducts with looping, multiple projections were analyzed including anterior-posterior (AP), left anterior oblique (LAO), and right anterior oblique (RAO) views with cranial and caudal angulation. The L2 was then measured using the best projection which best demonstrated the entire duct and its multiple complex turns.

The lower, median, and upper quartile values of the DCI in this patient cohort were used to classify the ducts into the following four classes: class I $(\leq 0.12)$, class II $(0.13-0.33)$, class III (0.34-0.44), and class IV ( $\geq 0.45)$ (Figure 2).

Associated pulmonary arterial stenosis (PAS) was documented. Total lower lobe index (TLLI), Nakata index and McGoon index were used as pulmonary parameters to estimate the growth of pulmonary arteries at closest time before the next planned surgery or intervention. 

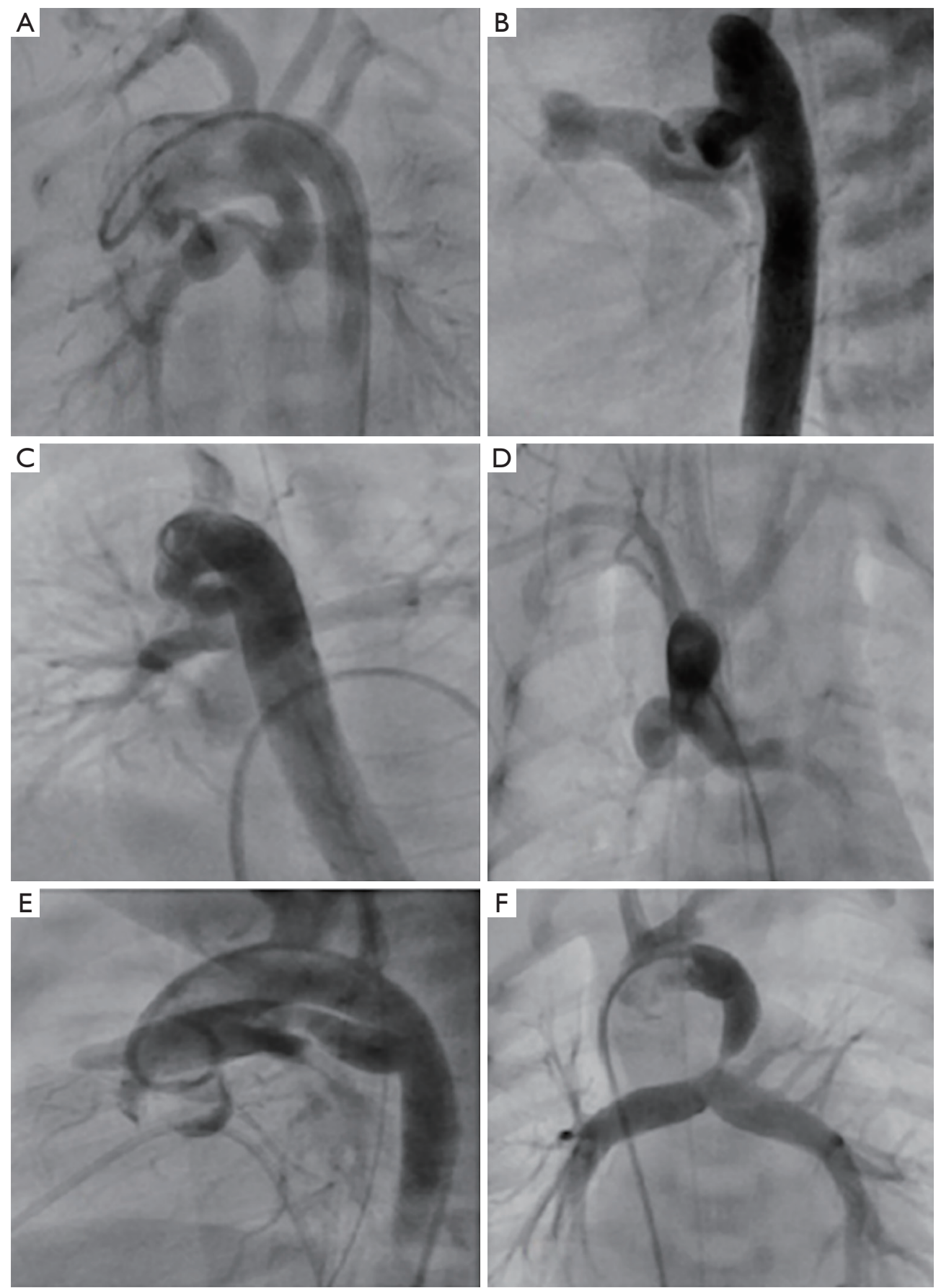

Figure 2 Demonstration of various ductal classes from the study patients. (A) Class III with ductal origin from underside of aortic arch; (B) class IV with origin from descending aorta; (C and D) class IV with ductal origin from underside of aortic arch; (E) class I with origin from descending aorta; (F) class II with origin from left brachiocephalic trunk.

\section{Statistical analysis}

All statistical analyses were performed using SPSS version 22. Continuous variables were reported as means \pm standard deviations (SD), and categorical variables as count (percentage). Non-paired Student's $t$-test was used to compare the means of continuous variables between 
the four different ductal morphology classes, while paired $t$-test was applied for comparing the means of pulmonary parameters before and after PDA stenting for the whole cohort. Chi square test was used for comparing categorical variables.

Odds ratios $(\mathrm{ORs}) \pm 95 \%$ confidence intervals $(95 \% \mathrm{CI})$ for the following parameters were calculated to assess any differences between the four ductal morphology classes: failure of stent-dependent survival (impaired outcome), need for PA arterioplasty, and need for unplanned surgery or RVOT-stent. A P value of 0.05 was set as the threshold for clinical significance. Kaplan-Meier survival curve of the four different classification classes was performed. The survival was defined according to the primary outcome i.e., arrival at the next planned surgery without death, permanent complications, or unplanned surgery/unplanned re-intervention in RVOT.

\section{Ethical statement}

The study complies with the Declaration of Helsinki (as revised in 2013). Owing to the purely retrospective study design, using available institutional clinical records, with an absence of impact on management of the patients included and completely anonymous data presentation, informed consent of the subjects (or their parents) and ethical approval have not been obtained.

\section{Results}

A total of 96 stents were implanted in 71 ducts for patients with DDPC. All stents were bare metal stents (BMS). The stenting was performed retrograde in $59(83.09 \%)$ and antegrade in $12(16.91 \%)$ patients. At the intervention, mean weight was $3.3 \mathrm{~kg}$ (SD 0.68), median 3.2 , range 3.2 $(2-5 \mathrm{~kg})$. Mean body surface area (BSA) was $0.20 \mathrm{~m}^{2}$ (SD $0.028)$, median 0.2 , range $0.14 \mathrm{~m}^{2}(0.14-0.18)$. Mean age was 18 days (SD 23.18) median 7 (range, 1-120 days).

No significant differences were found between the ductal morphology class and any of the following: hospital deaths, vascular access, and number of stents $(\mathrm{P}>0.05)$. Statistically significant differences were identified between the four classes in each of the following: primary outcome, need of unplanned surgery or unplanned PVperforation/RVOT-stent due to stent related inadequate pulmonary perfusion, pulmonary arterioplasty, origin of duct and in-stent re-intervention with $\mathrm{P}$ value $<0.05$ (Table 1).

\section{Mortality and complications}

No intervention-related mortality was reported in the catheterisation laboratory. Three hospital deaths (4.22\%) were reported 1, 7 and 47 days after the intervention (2 patients with class II, one with class IV). The first one was born in preterm twin pregnancy ( 30 gestational age) and died due to a septic shock. The second patient required an unplanned stent implantation in RVOT one week after duct stenting due to low oxygen saturation. Following the procedure, the patient was decompensated and died on ECMO. The third was born in preterm twin pregnancy ( 33 gestational age) and died in the intensive care unit (ICU) due to infant respiratory distress syndrome (IRDS) and multi-organ failure before reaching the next planned surgery. Five major complications (7.04\%) were reported; four in class IV and one in class I (Table 2). One patient had intervention-related permanent complications. This patient belonged to class IV and developed severe chronic neurologic complications associated with severe motoric retardation (Table 2). An impaired thrombophilia screening was reported in $40 \%$ of patients with in-stent thrombotic events. Three patients needed an emergency surgical shunt in the catheterisation laboratory during an in-stent re-intervention. Complete occlusion of the stent occurred while attempting to recanalize the stent in all of these patients; one of them needed ECMO.

\section{Primary and secondary outcome results}

The primary outcome in this study, defined earlier in the methods, was achieved in the majority of patients with DCI $<0.45$ (in class I, II, and III with $100 \%, 84.2 \%$, and $94.4 \%$ success rate respectively) compared to only $20 \%$ of those with DCI $\geq 0.45$ in class IV in which patients had very high risk of failure, odds ratio (OR) of 9 and $95 \%$ confidence interval (CI): 3.7-21.4. Need for unplanned surgery/PVP with RVOT-stent due to stent related insufficient pulmonary perfusion was reported in 13 patients, 10 (77\%) of which from class IV with OR 12.4 (95\% CI: 4-39), 2 (15.3\%) from class II, and one (7.7\%) from class III (Tables 1,3). A total of 9 patients (43.6\%) needed unplanned in-stent re-intervention (5 of them in class IV and 2 in III and II respectively). Need of pulmonary arterioplasty due to duct associated pulmonary stenosis was observed in 18 patients (25.3\% of total); eight in class IV with OR 3.3 (95\% CI: 1.5-7.1), six in class III, three in class II, and one in class I. Fifty-six patients completed the next planned procedure: $51.8 \%$ univentricular and $48.2 \%$ biventricular. Pulmonary parameters showed significant 
Table 1 Summarize of diagnosis, PDA characteristic and outcome in cohort study

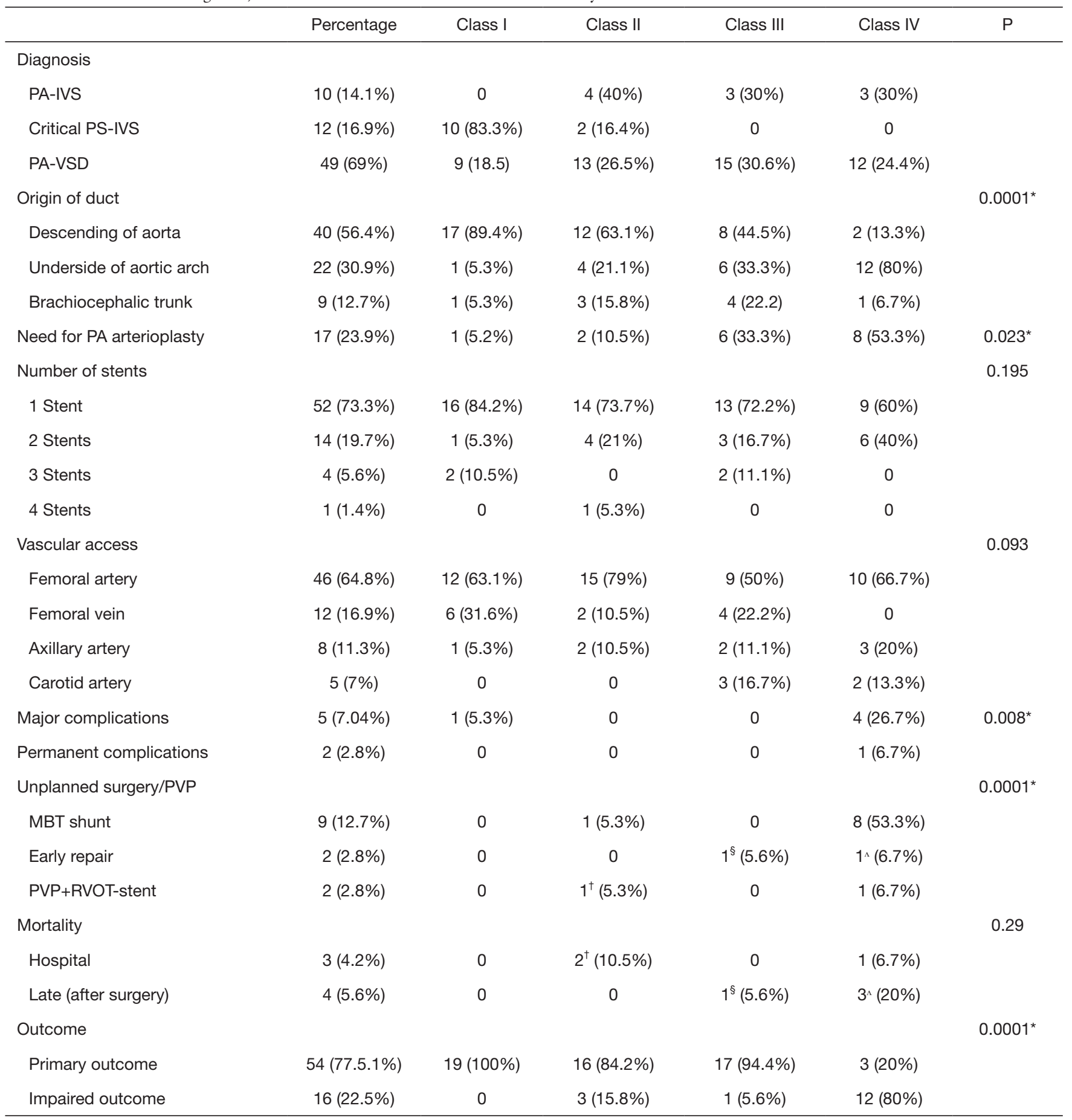

PA, pulmonary atresia; IVS, intact ventricular septum; VSD, ventricular septum defect; LPA, left pulmonary artery; RPA, right pulmonary artery. MBT Shunt: modified Blalock Taussig Shunt. PVP: pulmonary valve perforation. RVOT: right ventricular outflow tract, *P value $<0.05$ significant. ${ }^{\S}$ the same patient. ${ }^{\dagger}$ the same patient. ${ }^{\text {the same patient. }}$ 
Table 2 Intervention related Major complications

\begin{tabular}{|c|c|c|c|c|c|}
\hline & Patient 1 & Patient 2 & Patient 3 & Patient 4 & Patient 5 \\
\hline Origin of duct & Underside of a.a. & DAO & Underside of a.a. & TBC & Underside of a.a \\
\hline Vascular access & Fem. A & Fem. A & axil. A & Fem. A & axil. A \\
\hline $\mathrm{DCl}$ & 0.62 (IV) & $0(\mathrm{l})$ & 0.54 (IV) & $0.52(\mathrm{IV})$ & $0.62(\mathrm{IV})$ \\
\hline Thrombophilia screen & FFVLM & FFVLM & Not tested & Not tested & Not tested \\
\hline Outcome & MBT shunt & Glenn & Retardation & MBT shunt & MBT shunt \\
\hline
\end{tabular}

PA, pulmonary atresia; VSD, ventricular septum defect; IVS, intact ventricular septum; Underside of a.a., underside of aortic arch; DAO, descending aorta; TBC, truncus brachiocephalicus; DCI, ductal curvature index; axil. A, axillary artery; Fem. A, femoral artery; mBT, modified Blalock-Taussig shunt; S. Thrombosis, Stent thrombosis; FFVLM, familial factor V Leiden mutation.

Table 3 Need of unplanned surgery/unplanned RVOT stent



Stent in mm, stent in millimeter; PV-perforation, pulmonary valve perforation; $\mathrm{DCl}$, ductal curvature index.

increases in all of the 56 patients in whom the next planned procedure was achieved. Nakata index improved from a mean of 171 before to $286 \mathrm{~mm}^{2} / \mathrm{m}^{2}$ after stenting, McGoon index from 1.5 to 2.7 , and TLLI from 90 to $\left.169 \mathrm{~mm}^{2} / \mathrm{m}^{2}\right)(\mathrm{P}<0.001)$.

\section{Discussion}

Multiple formulas have been suggested to quantitatively measure the tortuosity of blood vessels in recent years (19) and this study adopted a formula of DCI that was previously used in a PDA-study (18). Calculating the DCI, we applied a ductal classification system on a cohort of patients with DDPC. The quartile approach was used to divide patients into four classes depending on their DCI-value, with the upper quartile threshold ( $\mathrm{DCI} \geq 0.45$ ) defining class IV; the group with the highest curvature index.

\section{High curvature index (class IV) and outcome}

While the majority of patients with DCI $<0.45$ achieved 


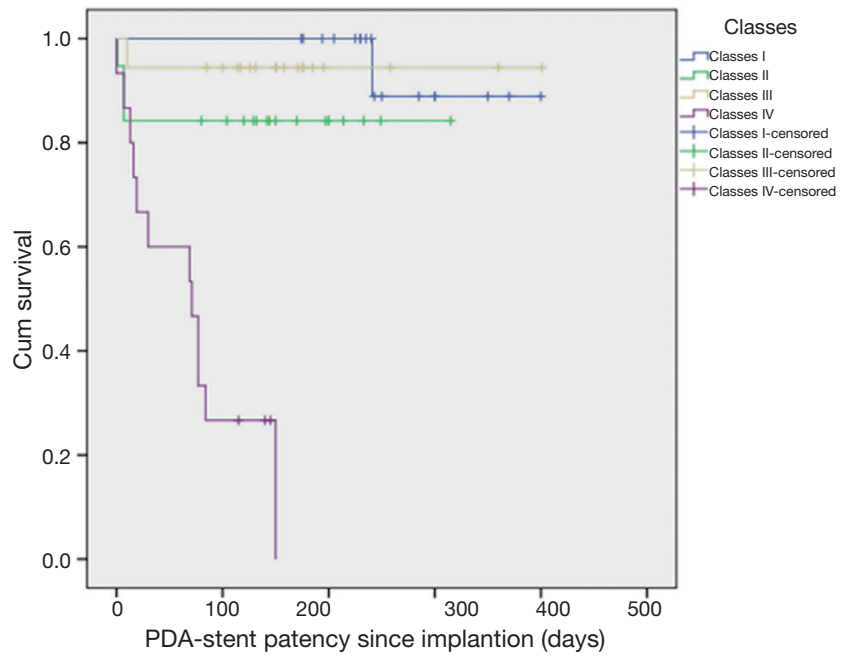

Figure 3 Kaplan-Meier curve: Probability of stent-dependent survival. Compared with class IV which showed very low survival, patients with class I-III had a much better survival rates with the majority reaching their next planned surgery. PDA: patent ductus arteriosus.

the primary outcome, only $20 \%$ of patients with DCI $\geq 0.45$ (class IV) achieved this endpoint in this study arriving uneventfully at their planned surgery. Class IV also, asymmetrically, represented more than $75 \%$ of all the cases that required unplanned surgery or unplanned PVP with RVOT-stent due to stent related inadequate pulmonary perfusion (Table 3). Additionally, almost half of all the patients requiring pulmonary arterioplasty due to duct associated pulmonary stenosis were found in class IV. Furthermore, all patients in this class needed either unplanned surgery/PVP and RVOT-stent or unplanned instent re-intervention. Early in coronary stent stenosis (ICS) and thrombosis, a phenomenon usually occurring in the elastic lamina, was highly observed in class IV of this cohort. Recoil and relocation of axially transmitted plaque have been suggested as potential causes of ICS $(20,21)$. These two factors can be induced and speeded up by increased tortuosity, deployment of long stent, and stent deformation as well as stent overexpansion and under-expansion (22) The need for in-stent reintervention in the present study, which was more frequent in ducts with low curvature index, may be explained by in-stent restenosis, which can take place weeks or even months after the intervention due to thrombogenesis, neointima formation, and remodelling $(20,21)$. We found that stent deformation and malposition were more often observed in patients whose ducts had increased DCI (class IV) making the in-stent reintervention a high-risk procedure in these patients.

The survival analysis demonstrated a clear and early dissociation between class IV and the rest of the classes with patients in the former group experiencing a sharp and mostly very acute outcome failure (Figure 3). Patients with DCI $<0.45$, however, had a much better survival rate with the majority reaching their next planned surgery.

Although some studies have demonstrated the safety and efficacy of duct stenting as an alternative to a surgical creation of mBT Shunt $(8,23,24)$ very few have assessed the impact of ductal morphology on the duct stenting outcome $(25,26)$. Qureshi et al. in 2019 described a new ductal morphological scheme based on ductal tortuosity classifying the ducts into three types: type $\mathrm{I}=$ straight, type II = one curve, and type III = multiple complex turns (18). Their results showed a significantly higher rate of pulmonary arterioplasty and unplanned reintervention in patients with type III. The latter observation is in agreement with the results of the present study that demonstrated a significantly greater need for pulmonary arterioplasy in patients with increased curvature Index (class IV). Comparison between the two cohorts was summarised in Table 4 .

Although the mentioned study calculated the DCI and found it to be correlated with the tortuosity type, no cutoff values were provided for estimating the severity of the DCI in the previous report. In our cohort, we proposed a quantitative cut-off threshold ( $\mathrm{DCI} \geq 0.45$ ) to estimate highrisk PDA-stenting.

Three-dimensional computed tomography (3D CT) and 3D echocardiography may offer more accurate calculation of the curvature index and should be considered in future studies.

A good understanding of the duct morphology and a correct selection of the best suitable vascular access based on the duct origin as well as the use of an appropriate softtipped guide-wire, which does not alter the ducts native course too much, but gives enough support to safely advance the stent [e.g., Mailman, comp], are essential to optimise the stenting outcome and minimize major complications. Also wire retraction is a critical moment, because of the soft ductal tissue. The use of a long sheath can help to stabilize the stent, while retrieving the wire.

It is also worth noting that a significant proportion of patients with thrombotic events in this cohort were found to have abnormal thrombophilic tests. Thrombophilia screening is, therefore, highly recommended prior to stenting to minimize thrombotic events especially in high 
Table 4 Distribution of ductal morphology: ductal curvature index classification versus ductal tortuosity index scheme

\begin{tabular}{lccccc}
\hline \multirow{2}{*}{ TI } & \multicolumn{3}{c}{ DCI } & Class IV & Total \\
\cline { 2 - 5 } Type I & Class I & Class II & Class III & 0 & 19 \\
Type II & $19(100 \%)$ & 0 & 0 & $3(20 \%)$ & 39 \\
Type III & 0 & $19(100 \%)$ & $17(94.4 \%)$ & $1(5.5 \%)$ & $12(80 \%)$ \\
\hline
\end{tabular}

TI, tortuosity index; DCl, ductal curvature index.

risk patients.

Limitations: as a retrospective observational study potentially important secondary outcome measures could not be fully assessed. The single-center data collection limits the total number of patients to be recruited and reflects the ambitions and experiences of only a small number of interventionalists. Additionally, all stents used in this cohort were bare metal stents (BMS) and the results may not be applicable to drug eluted stents.

\section{Conclusions}

Ducts with a high ductal curvature index are associated with significant complications and are at higher risk to fail stenting compared to ducts with a lower curvature index. This study suggests that a clinical cut-off point based on the severity of ductal curvature may well identify high risk patients for stenting in whom the duct stenting may be insufficient to bridge them to the next planed surgery or intervention. The results indicate that class IV (DCI $\geq 0.45$ ), represents a truly high-risk group of patients with unfavorable stenting outcome. In contrast, patients with curvature index lower than 0.45 , which represents $75 \%$ of total cohort, have very satisfactory outcomes. A prospective study is needed to validate these results and see if the new curvature-based classification system can perform similarly in different cohorts.

\section{Acknowledgments}

Funding: None.

\section{Footnote}

Reporting Checklist: The authors have completed the STROBE reporting checklist. Available at http://dx.doi. org/10.21037/tp-21-17
Data Sharing Statement: Available at http://dx.doi. org/10.21037/tp-21-17

Conflicts of Interest: All authors have completed the ICMJE uniform disclosure form (Available at http://dx.doi. org/10.21037/tp-21-17). The authors have no conflicts of interest to declare.

Ethical Statement: The authors are accountable for all aspects of the work in ensuring that questions related to the accuracy or integrity of any part of the work are appropriately investigated and resolved. The study complies with the Declaration of Helsinki (as revised in 2013). Owing to the purely retrospective study design, using available institutional clinical records, with an absence of impact on management of the patients included and completely anonymous data presentation, informed consent of the subjects (or their parents) and ethical approval have not been obtained.

Open Access Statement: This is an Open Access article distributed in accordance with the Creative Commons Attribution-NonCommercial-NoDerivs 4.0 International License (CC BY-NC-ND 4.0), which permits the noncommercial replication and distribution of the article with the strict proviso that no changes or edits are made and the original work is properly cited (including links to both the formal publication through the relevant DOI and the license). See: https://creativecommons.org/licenses/by-nc-nd/4.0/.

\section{References}

1. Balasubramanya S, Zurakowski D, Borisuk M, et al. Right ventricular outflow tract reintervention after primary tetralogy of Fallot repair in neonates and young infants. J Thorac Cardiovasc Surg 2018;155:726-34.

2. Curzon CL, Milford-Beland S, Li JS, et al. Cardiac 
surgery in infants with low birth weight is associated with increased mortality: analysis of the Society of Thoracic Surgeons Congenital Heart Database. J Thorac Cardiovasc Surg 2008;135:546-51.

3. Lechner E, Wiesinger-Eidenberger G, Weissensteiner $\mathrm{M}$, et al. Open-heart surgery in premature and lowbirth-weight infants--a single-centre experience. Eur J Cardiothorac Surg 2009;36:986-91.

4. Hickey EJ, Nosikova Y, Zhang H, et al. Very low-birthweight infants with congenital cardiac lesions: is there merit in delaying intervention to permit growth and maturation? J Thorac Cardiovasc Surg 2012;143:126-36, 36 e1.

5. Azakie A, Johnson NC, Anagnostopoulos PV, et al. Cardiac surgery in low birth weight infants: current outcomes. Interact Cardiovasc Thorac Surg 2011;12:40913, discussion 14.

6. Schneider M, Zartner P, Sidiropoulos A, et al. Stent implantation of the arterial duct in newborns with ductdependent circulation. Eur Heart J 1998;19:1401-9.

7. Schranz D, Michel-Behnke I, Heyer R, et al. Stent implantation of the arterial duct in newborns with a truly duct-dependent pulmonary circulation: a single-center experience with emphasis on aspects of the interventional technique. J Interv Cardiol 2010;23:581-8.

8. Glatz AC, Petit CJ, Goldstein BH, et al. Comparison Between Patent Ductus Arteriosus Stent and Modified Blalock-Taussig Shunt as Palliation for Infants With Ductal-Dependent Pulmonary Blood Flow: Insights From the Congenital Catheterization Research Collaborative. Circulation 2018;137:589-601.

9. Bentham JR, Zava NK, Harrison WJ, et al. Duct Stenting Versus Modified Blalock-Taussig Shunt in Neonates With Duct-Dependent Pulmonary Blood Flow: Associations With Clinical Outcomes in a Multicenter National Study. Circulation 2018;137:581-8.

10. Santoro G, Capozzi G, Caianiello G, et al. Pulmonary artery growth after palliation of congenital heart disease with duct-dependent pulmonary circulation: arterial duct stenting versus surgical shunt. J Am Coll Cardiol 2009;54:2180-6.

11. Li JS, Yow E, Berezny KY, Rhodes JF, et al. Clinical outcomes of palliative surgery including a systemic-topulmonary artery shunt in infants with cyanotic congenital heart disease: does aspirin make a difference? Circulation 2007;116:293-7.

12. Aggarwal V, Dhillon GS, Penny DJ, et al. Drug-Eluting Stents Compared With Bare Metal Stents for Stenting the Ductus Arteriosus in Infants With Ductal-Dependent Pulmonary Blood Flow. Am J Cardiol 2019;124:952-9.

13. Boucek DM, Qureshi AM, Goldstein BH, et al. BlalockTaussig shunt versus patent ductus arteriosus stent as first palliation for ductal-dependent pulmonary circulation lesions: A review of the literature. Congenit Heart Dis 2019;14:105-9.

14. Lee KJ, Hinek A, Chaturvedi RR, Almeida CL, et al. Rapamycin-eluting stents in the arterial duct: experimental observations in the pig model. Circulation 2009;119:2078-85.

15. Santoro G, Gaio G, Palladino MT, et al. Arterial duct stenting: Do we still need surgical shunt in congenital heart malformations with duct-dependent pulmonary circulation? J Cardiovasc Med (Hagerstown) 2010;11:852-7.

16. Santoro G, Gaio G, Palladino MT, et al. Stenting of the arterial duct in newborns with duct-dependent pulmonary circulation. Heart 2008;94:925-9.

17. Alwi M, Mood MC. Stenting of Lesions in Patent Ductus Arteriosus with Duct-Dependent Pulmonary Blood Flow: Focus on Case Selection, Techniques and Outcome. Interv Cardiol Clin 2013;2:93-113.

18. Qureshi AM, Goldstein BH, Glatz AC, et al. Classification scheme for ductal morphology in cyanotic patients with ductal dependent pulmonary blood flow and association with outcomes of patent ductus arteriosus stenting. Catheter Cardiovasc Interv 2019;93:933-43.

19. Han HC. Twisted blood vessels: symptoms, etiology and biomechanical mechanisms. J Vasc Res 2012;49:185-97.

20. Alraies MC, Darmoch F, Tummala R, et al. Diagnosis and management challenges of in-stent restenosis in coronary arteries. World J Cardiol 2017;9:640-51.

21. Bennett MR. In-stent stenosis: pathology and implications for the development of drug eluting stents. Heart 2003;89:218-24.

22. Buccheri D, Piraino D, Andolina G, et al. Understanding and managing in-stent restenosis: a review of clinical data, from pathogenesis to treatment. J Thorac Dis 2016;8:E1150-62.

23. Alwi M, Choo KK, Latiff HA, Kandavello G, et al. Initial results and medium-term follow-up of stent implantation of patent ductus arteriosus in duct-dependent pulmonary circulation. J Am Coll Cardiol 2004;44:438-45.

24. Gewillig M, Boshoff DE, Dens J, et al. Stenting the neonatal arterial duct in duct-dependent pulmonary circulation: new techniques, better results. J Am Coll Cardiol 2004;43:107-12. 
25. Alwi M. Stenting the ductus arteriosus: Case selection, technique and possible complications. Ann Pediatr Cardiol 2008;1:38-45.

26. Jadhav SP, Aggarwal V, Masand PM, et al. Correlation

Cite this article as: Mini N, Schneider MBE, Zartner PA. Use of the ductal curvature index to assess the risk of ductal stenting in patients with duct-dependent pulmonary circulation. Transl Pediatr 2021;10(5):1307-1316. doi: 10.21037/tp-21-17 of ductus arteriosus length and morphology between computed tomographic angiography and catheter angiography and their relation to ductal stent length. Pediatr Radiol 2020;50:800-9. 